\title{
Particle Physics for Primary Schools - enthusing future Physicists
}

\section{Cristina Lazzeroni}

University of Birmingham

School of Physics and Astronomy, Edgbaston B15 2TT, UK

E-mail: c.lazzeroni@bham.ac.uk

\section{Maria Pavlidou $^{1}$}

University of Birmingham

School of Physics and Astronomy, Edgbaston B15 2TT, UK

E-mail: m.pavlidou@bham.ac.uk

Recently the realisation that children make decisions and choices about subjects they like during their primary school years became widely understood. For this reason academic establishments have started to include activities aimed at the younger ages in their portofolio. During the last academic year we designed and trialled a particle physics workshop for primary schools. The workshop allows young children (ages 8-11) to learn the world of particles, use creative design to make particle models and engage in creative writing to describe how particles interact with each other. The workshop has been trialled in local primary schools, receiving positive evaluation. The resources were improved, based on the feedback given by the primary school teachers.

\section{${ }^{1}$ Speaker}




\section{Introduction}

The particle physics workshop explores the ability of young children to be imaginative and creative, and exploits it to teach them the fundamentals of particle physics in a fun way.

\section{Targeting younger ages}

The world of fundamental particles shares familiar concepts with those of every day's life: the fundamental particles of the standard model can be grouped into three families: the quarks, the leptons, and the bosons. Moreover, particle interactions could be loosely described as governed by the "likes" and "dislikes" between the particles. Such general ideas are accessible to young children because they understand how families and friendships work in their social groups.

Recent studies show that children make up their minds about career-paths and the subjects they like earlier than secondary school [1]. This means that to mitigate some of the biases and misconceptions regarding physics, and specifically particle physics, we should enthuse and inform children when they are still in primary education. In addition, discoveries in particle physics are often hugely popular in the news and reach a very broad audience, including families with children. By nature young children are inquisitive and want to know more about topics that are not typically discussed in schools, such as particle physics. The thirst of knowledge and the desire to understand the basic ideas of modern physics is a drive that educators and researchers can exploit. Another interesting aspect is that stereotypes such as "physics is for boys" appear to be stronger the older the students are. Therefore one can argue that the battle of attracting more females towards physics is harder in secondary schools and easier in primary schools where the girls have not been diverted from physics by peer pressure or other social conventions. From our own experience in all the primary schools we worked with, all children approached the workshop with an open mind and an enthusiastic involvement regardless of their gender.

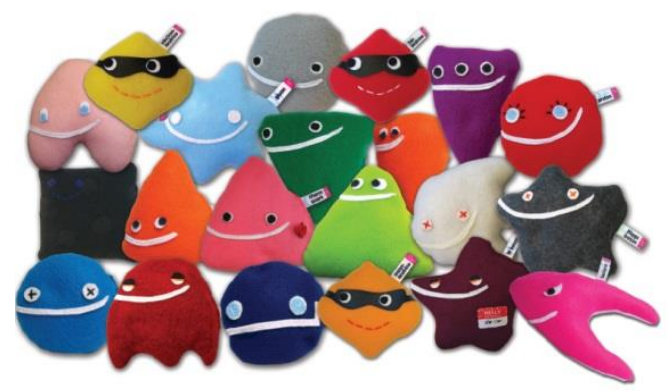

Figure 1 . The particle zoo fluffy toys [2]
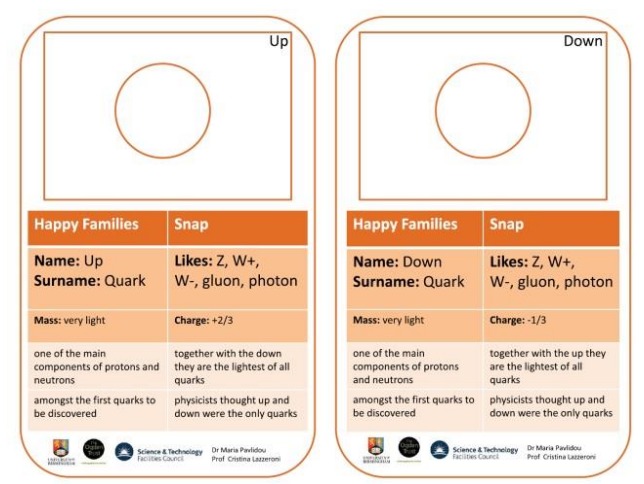

Figure 2. Trump cards which include some of the properties of fundamental particles

\section{Tools for successful learning}

In developing the workshop we used three main learning tools: visualisation of the particles using the particle zoo fluffy toys (see Figure 1), game playing using appropriately designed trump-cards (see Figure 2) and freedom of expression in the creative parts of the workshop.

To mitigate the abstractness of particle physics and its concepts, we introduce the fundamental particles and their properties using the particle zoo fluffy toys; this allows children to create a "personality" for each particle in their minds. In the particle zoo each particle has been designed so that its appearance links to a specific property. For example the strange quark 
has three eyes and the neutrinos have masks which hint towards their elusive nature. In this way children can access the new ideas and concepts in a more direct and practical way.

The importance of learning through playing has been documented in many research studies [3]. Two favourite and well-known card games that are commonly used in the UK are Happy Families and Snap. In the particle physics workshop, the Happy Families game is used as a helping tool in embedding the names of the particles and the "families" they belong to into the children's minds. Each family has its own designated colour in the cards, whereas the family name appears as the "surname" of the particle. Antimatter families share the same colour as the equivalent matter families to show that these are strongly linked together. The Snap game is used for recognising "likes and "dislikes" between particles which is a good introduction to particle interaction. The idea that particles interact only with others they like is easily understood since children can related it to their own behaviour.

An important aspect of the learning process in the particle physics workshop is the creativity and freedom that is given to the students when they are asked to design their own particles and to describe particle interaction using a range of media such as story telling, dramatisation, cartoon design and many more [4]. We have been impressed by the quality and variety of creative work we have seen over the years on particle designs (see Figure 3 ) and on describing particle interactions (see Figure 4). From poems and songs to acting and dancing, students choose to express themselves in ways that reflect their personality, aptitudes and strenghts. This results in a very positive student experience where the talents of individuals shine throughout the day.

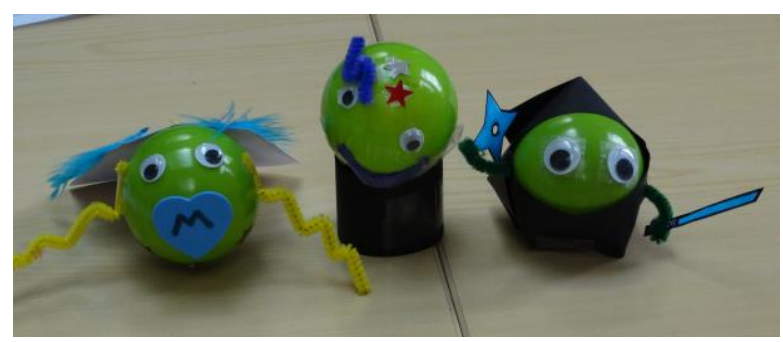

Figure 3. Leptons where designs are inspired by their properties

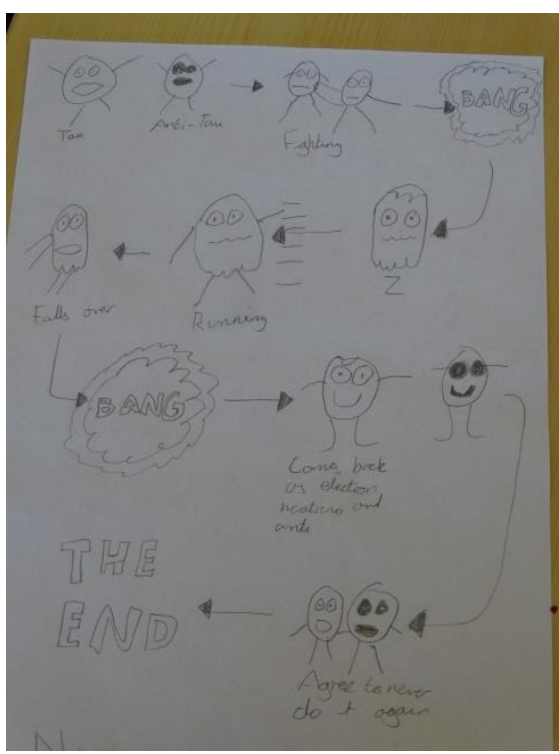

Figure 4. Cartoon drawn by students, describing particle interaction

\subsection{Delivering concepts in an accessible way}

The particle physics workshop focuses on teaching students how physicists test and broaden their understanding, what happens at CERN and the role of particle detectors and accelerators [5]. In dealing with these concepts, we use everyday examples that children are familiar with; the LHC is presented as a "motorway" for particles and the ATLAS detector as a very fast digital camera able to take millions of photographs in the blink of an eye. We use the image that in particle collisions the nature of protons is investigated as with a very powerful microscope, revealing the presence of the smallest "fundamental" particles. To tackle the 
concept of matter and antimatter we link these to the idea of identical twins that differ only in one characteristic. The concepts of creation and annihilation are introduced as events in the particle-world, where particles can appear and disappear or turn into other particles. In our experience children are gladly willing to accept these new concepts, and elaborate on them.

\section{Evaluating short and long term impact}

The workshop so far has engaged around 600 primary school children. Our resources have been translated into Greek and we have trained other University and research/outreach professionals on how to use them [6]. In the latest of our events we systematically collected evaluation from students and teachers to measure the short and long term impact. Immediately after the end of the workshop students were asked to fill in a short questionnaire which records their newly acquired knowledge, their desire to share it with friends and family and their attitudes towards studying physics further. A similar questionnaire was given to the teachers for the purpose of recording their own knowledge and enthusiasm for the subject as well as their plans to make a display of the particle world in their classroom. We also leave a second questionnaire with the teacher, with the request to allow the same students to complete it a month later. Evaluation of the teacher questionnaire and comparison of the two student questionnaires has allowed us to analyse the short and long term impact of the workshop.

From the evaluation questionnaires we have collected so far, it is clear that all students were able to describe at least one piece of the newly acquired knowledge a month after they attended the workshop. A very high number of students (93\%) went home and explained what they learned to friends and family. A similar number of students admitted that they are more interested in science as a result of attending the workshop. About $80 \%$ of the students said that the day made them more excited about studying physics further.

The results were somehow less encouraging regarding the responses of the teachers. Only $25 \%$ of the teachers made a display of the particle world in their classroom, although all of them admitted that their students would benefit from a similar day next year. Moreover, only half of the teachers said that they will use the resources of the particle physics workshop in future years. This feedback might relate to the lack of specialised knowledge in the teaching profession in primary schools. In the UK, a large number of primary school teachers do not have a scientific background. This can lead to a lack confidence in teaching the difficult concepts of modern physics. In order to tackle this problem, we co-organised the first ever training course for primary school teachers in the UK at CERN called "playing with protons UK" [7], with the support of The Ogden Trust and the EU CREATIONS project.

\section{Conclusions}

For many years the most recent discoveries relating to particle physics were presented only to secondary school students studying A level physics. More recently, it has become clear that children make decisions about their interests and future careers while they are in primary school. This, in combination with the urge to encourage more girls towards physics, was the driving force behind creating a tailor-made workshop on particle physics for primary schools.

The workshop uses creative approaches to learning, which is a powerful tool in enthusing young students towards even abstract subjects. The method of learning through playing is used in the workshop and there is good evidence suggesting that the acquired knowledge is sustained for longer periods of time.

We conclude that primary school children should be allowed to engage with cutting-edge modern physics since the exposure aids motivation in tackling the perceived difficulty of the subject and enhances the understanding of the current challenges scientists face in today's world. Finally, the importance of training primary school teachers in modern physics topics must come to the forefront of the efforts to improve primary school education and to support the choices of future generations towards studying physics. 


\section{References}

[1] van Tuijl, C. \& van der Molen, J.H.W. Study choice and career development in STEM fields: an overview and integration of the research, Int J Technol Des Educ (2016) 26: 159. doi:10.1007/s10798-015-9308-1

[2] https://www.particlezoo.net/ (accessed on 31 August 2017)

[3] Honey, M. \& Kanter, D. E. Design, Make, Play: Growing the Next Generation of STEM Innovators, 1st ed. New York [u.a.]: Routledge, 2013. Print.

[4] Root-Bernstein, Arts and crafts as adjuncts to STEM education to foster creativity in gifted and talented students, R. Asia Pacific Educ. Rev. (2015) 16: 203. doi:10.1007/s12564-015-9362-0

[5] Pavlidou, M \& Lazzeroni, C. Particle physics for primary schools-enthusing future physicists, 2016 Phys. Educ. 51054003

[6] http://www.birmingham.ac.uk/schools/physics/outreach/Primary-

Schools/MiniParticlePhysicsWorkshops.aspx (accessed on 1 September 2017)

[7] https://indico.cern.ch/event/615287/timetable/ (accessed on 1 September 2017) 